\title{
GINECOSMASTIA INDUCIDA POR EFAVIRENZ EN ADOLESCENTES. SERIE DE CASOS.
}

\section{Gynecosmastia induced efavirenz in teens. Case series. \\ *Karen Erazo}

\section{RESUMEN}

Antecedentes: La calidad de vida y el pronóstico de los pacientes con infección por el virus de inmunodeficiencia humana $(\mathrm{VIH})$ ha mejorado enormemente desde la introducción de la terapia antirretroviral de gran actividad (TARGA). Sin embargo se han descrito numerosos efectos adversos y limitaciones en cuanto a la tolerabilidad como resultado del uso crónico de los mismos, esto es sin duda uno de los mayores retos y preocupaciones para el profesional de salud encargado del cuidado de estos pacientes. Lipomastia (pseudoginecomastia), una ampliación del pecho debido a la adiposidad central, puede ocurrir como parte de un síndrome de redistribución de la grasa que se ha asociado con los regímenes TARGA. Se presenta una serie de cuatro casos de pacientes con diagnóstico de ginecomastia asociada con regímenes de TARGA basado en efavirenz. Todos los casos alcanzaron respuestas inmunológicas y virológicas con éxito a TARGA y la aparición de la ginecomastia se presentó en diferentes etapas de la adolescencia. En todos los casos los pacientes tenían más de 3 años de uso del medicamento observándose regresión de la ginecomastia después de suspender el efavirenz (período de 6 meses promedio). En conclusión, pensamos que la ginecomastia en pacientes adolescentes con VIH en TARGA se debe sos-

*Médico Pediatra del Centro de Atención Integral, Hospital Dr Mario Catarino Rivas, Docente de la UNAH-VS.

Dirigir correspondencia a: karenerazo@yahoo.com.

Recibido: 20 noviembre $2014 \quad$ Aprobado: 13 de abril 2015. pechar en quienes están recibiendo regímenes conteniendo efavirenz.

\section{PALABRAS CLAVE}

Antirretrovirales, Efectos Colaterales, Ginecomastia y reacciones adversas relacionadas con medicamentos.

\section{ABSTRACT}

Background: The quality of life and prognosis of patients infected with human immunodeficiency virus (HIV) have improved dramatically since the introduction of antiretroviral therapy (ART). However described numerous adverse effects and limitations in terms of tolerability as a result of chronic use of them, this is definitely one of the biggest challenges and concerns for the health professional responsible for the care of these patients. Lipomastia (pseudoginecomastia), a breast enlargement due to central adiposity, may occur as part of a syndrome of fat redistribution that has been associated with ART regimens. A series of four cases of patients diagnosed with gynecomastia associated with efavirenz-based ART regimens is presented. All cases reached immunologic and virologic responses to ART successfully and the appearance of gynecomastia was presented at different stages of adolescence. In all cases the patients had more than three years of medication use regression of gynecomastia observed after discontinuation of efavirenz (average period of 6 months). In conclusion, we think the gynecomastia in adolescent patients with 
HIV on HAART should be suspected in those receiving efavirenz-containing regimens.

\section{KEYWORDS}

Anti-retroviral Agents, Drug-Related Side Effects, Gynecomastia and Adverse Reactions.

\section{INTRODUCCION}

La ginecomastia auténtica se define como un aumento del tamaño de la mama en el varón debido a la proliferación del tejido glandular ductal y de estroma periductal. La mitad o más de los muchachos adolescentes tienen algún desarrollo de los senos durante la pubertad, esto puede surgir como un estado anormal asociado con trastornos o enfermedades metabólicas, como resultado de la disminución natural de la producción de testosterona en los hombres mayores o como un efecto secundario de alguna medicación, esto último puede ser responsable de la ginecomastia entre un 10 y un $25 \%$ de los casos. ${ }^{(1)}$ En los varones, la condición es a menudo una fuente de sufrimiento psicológico, sin embargo, el $75 \%$ de los casos de ginecomastia puberal resuelven en los dos años siguientes al inicio sin necesidad de tratamiento. ${ }^{(2)}$ Esta entidad no debe confundirse con Lipomastia (pseudoginecomastia) o acumulación de grasa mamaria que constituye una de las alteraciones morfológicas integradas en el síndrome de lipodistrofia. La ginecomastia tiene un pico trimodal de incidencia y frecuencia que se presenta en los recién nacidos, adolescentes y hombres mayores de 50 años de edad. Las estimaciones de prevalencia de ginecomastia detectable en los adolescentes han sido tan altas como $64 \%$, pero pueden incluir casos de pseudoginecomastia. ${ }^{(3)}$
Los medicamentos que han sido implicados en el desarrollo de ginecomastia, actúan por diferentes mecanismos fisiopatológicos no siempre bien conocidos. Algunos actúan en forma directa sobre el receptor de estrógenos en la mama, otros por alteraciones en el metabolismo de los esteroides o interferencia en la unión con su proteína transportadora, lo cual aumenta especialmente la concentración de estrógenos libres. ${ }^{(4)}$ Están implicados antiandrógenos (flutamida), antimicrobianos (isoniacida, ketoconazol, metronidazol, griseofluvina, D-penicilamina), antiulcerosos (cimetidina, ranitidina, omeprazol), citotóxicos (alquilantes,vincristina, nitrosureas, metotrexato), medicamentos cardiovasculares (espironolactona, IECA, amiodarona, bloqueadores de los canales de calcio, digoxina, metildopa, reserpina), hormonas (andrógenos, esteroides anabolizantes, gonadotropina coriónica, estrógenos, hormona de crecimiento), antirretrovirales (efavirenz, algunos inhibidores de proteasa como Indinavir, saquinavir, ritonavir nelfinavir), psicofármacos (fenotiacinas), alopurinol, drogas de abuso (alcohol, anfetaminas, heroína, metadona, marihuana) y plantas medicinales (aceite de lavanda, aceite de té verde). La ginecomastia inducida por medicamentos suele ser bilateral, pero a menudo es asimétrica y, en ocasiones, dolorosa. Se relaciona tanto con la dosis diaria como con la duración del tratamiento. Se han descrito períodos de inducción muy variables, incluso de años desde el inicio del tratamiento. Suele ser reversible, aunque la recuperación completa puede tardar meses después de la retirada del medicamento sospechoso. ${ }^{(5)}$

Pseudoginecomastia ha sido reportado en personas infectados por el VIH que toman 
tratamiento antirretroviral (TAR) como parte del síndrome de redistribución de la grasa. ${ }^{(6)}$ Efavirenz (EFV) es uno de los medicamentos antirretrovirales más recetados en primera línea de tratamiento para la infección por VIH. Es el único no nucleósido que se recomienda actualmente como terapia preferente en personas que no han tomado antes medicación y su dosis es muy cómoda, con una pastilla una vez al día. Varios estudios han mostrado que es tan eficaz como los inhibidores de la proteasa cuando se administra con una base de dos nucleósidos, incluso frente a altas carga virales iniciales. Su perfil tóxico estaba más o menos bien caracterizado: efectos adversos en el sistema nervioso central especialmente durante las primeras ocho semanas, aunque no sólo; posible pero en general ligera elevación de triglicéridos; algo de exantema y casos de ginecomastia en varones. ${ }^{(7)}$ Durante mucho tiempo se consideró que el EFV no estaba asociado a problemas de lipodistrofia en personas con $\mathrm{VIH}$, pero esto en la actualidad ha cambiado. Se han publicado estudios dedicados exclusivamente a las complicaciones metabólicas y cardiovasculares de la terapia antirretroviral, cuyos resultados indican que tomar efavirenz supone un mayor riesgo de desarrollo de lipoatrofia (pérdida de grasa subcutánea en las extremidades) que lopinavir/ritonavir. ${ }^{(8)}$

En Honduras el tratamiento antirretroviral de primera línea usado es la combinación de zidovudina, lamivudina y efavirenz. ${ }^{(9)}$ En el Centro de Atención Integral Pediátrico del Hospital Dr. Mario Catarino Rivas en los últimos 2 años se ha observado la aparición de ginecomastia en 4 pacientes masculinos con esta terapia de primera línea por más de 2 años, lo que nos lleva a reportar esta serie de casos y realizar una revisión bibliográfica del tema.

\section{Caso No. 1}

Masculino de 20 años con diagnóstico de $\mathrm{VIH}$ positivo desde los 8 años de edad, con vía de transmisión perinatal desde su diagnóstico en TAR con terapia de primera línea zidovudina, lamivudina y efavirenz, con cargas virales no detectables desde el 2005, con último control de CD4 de 480 células. A los 11 años y con 3 años de uso de antirretrovirales presenta ginecomastia bilateral de predominio derecho, dolorosa sin descarga canalicular por los pezones la cual persiste y aumenta de tamaño por lo que a los 15 años con 8 meses se decide retirar Efavirenz sustituyéndolo por Lopinavir/Ritonavir. Resolución de la ginecomastia 3 años después del retiro del Efavirenz.

\section{Caso No. 2}

Masculino de 17 años de edad, con diagnóstico de VIH positivo desde los 9 años de edad, con vía de transmisión perinatal, en TAR desde su diagnóstico con terapia de primera línea Zidovudina, Lamivudina y Efavirenz, con cargas virales no detectables en los controles posteriores al inicio de la terapia, último conteo de CD4 de 830 células. A los 15 años de edad con 6 años de uso de antirretrovirales presenta ginecomastia bilateral levemente dolorosa, se mantiene en vigilancia y debido a que progresa de tamaño un año después se retira el Efavirenz sustituyéndolo por Lopinavir/Ritonavir. Persiste con la ginecomastia, en la actualidad sin cambios.

\section{Caso No. 3}

Masculino de 13 años, con diagnóstico de 
VIH positivo desde los 2 años de edad, con vía de transmisión perinatal, desde su diagnóstico con TAR de primera línea Zidovudina, Lamivudina y Efavirenz, con cargas virales no detectables en los controles posteriores al inicio de la terapia, último conteo de CD4 de 642 células. A los 12 años de edad con 10 años de uso de antirretrovirales presenta ginecomastia unilateral derecha levemente dolorosa, se retira el Efavirenz sustituyéndolo por Lopinavir/Ritonavir. Persiste con la ginecomastia en la actualidad sin cambios.

\section{Caso No. 4}

Masculino de 15 años, con diagnóstico de VIH positivo desde los 3 años de edad, con vía de transmisión perinatal, desde su diagnóstico con TAR de primera línea Zidovudina, Lamivudina y Efavirenz, con cargas virales no detectables en los controles posteriores al inicio de la terapia, último conteo de CD4 de 1652 células. A los 14 años de edad con 11 años de uso de antirretrovirales presenta ginecomastia unilateral derecha no dolorosa, se retira el Efavirenz sustituyéndolo por Lopinavir/Ritonavir. Persiste con la ginecomastia en la actualidad sin cambios.

\section{DISCUSIÓN}

TARGA consiste en tres o más combinaciones de antirretrovirales. Los antirretrovirales se clasifican de acuerdo a la fase de ciclo vital de los retrovirus que inhiben. Comúnmente inhibidores de la transcriptasa inversa, análogos de nucleósidos con inhibidores de la transcriptasa inversa, no análogos de nucleósidos o inhibidores de la proteasa son utilizados como esquemas de primera línea. El principal objetivo de la TARGA es disminuir la carga viral en los pacientes con infección por el VIH. ${ }^{(10)}$ Los cuatro pacientes presentados en esta serie de casos su vía de infección fue la perinatal y mantenían cargas virales no detectables o menos de 20 copias. Con terapia antirretroviral de primera línea zidovudina, lamivudina y efavirenz.

Con la introducción de la TARGA, muchos efectos secundarios han sido descritos, la ocurrencia de los mismos puede interferir con la adherencia terapéutica y el pronóstico a largo plazo y por tal razón requiere atención. La ginecomastia es uno de ellos, observándose en el $2.8 \%$ en pacientes con más de dos años de tratamiento antirretroviral. ${ }^{(11)}$ Ginecomastia prepuberal es una condición rara y más frecuentemente clasificada como idiopática. En adultos infectados por el $\mathrm{VIH}$ ginecomastia es un efecto secundario reconocido pero poco frecuente de tratamiento antirretroviral y en su mayoría atribuido al uso de Efavirenz. ${ }^{(12-14)}$ Una hipótesis del mecanismo productor es que la restauración inmunológica debido a la supresión viral, hace que el TARGA mejore la respuesta de citoquinas $T$ auxiliar que mejora la disponibilidad de estrógeno produciendo ginecomastia. ${ }^{(15)}$ Sikora y sus colegas demostraron la unión directa del Efavirenz al receptor de estrógeno alfa de mama. Este receptor induce el crecimiento celular en 17 $\beta$ modelo de cáncer de mama dependiente de estradiol. ${ }^{(16)}$

En un estudio realizado en Europa se demostró una incidencia similar del 2,9\% (15 de cada 516 pacientes) en los pacientes masculinos tratados con diferentes regímenes de antirretrovirales. ${ }^{(17)}$ Manfredi y sus colegas describen tres casos de ginecomastia confirmado por la ecografía en niños 
a partir de 11 años (1) y 12 años (2) con infección por el VIH adquirida por vía perinatal. ${ }^{(12,18)}$ Estos niños estaban tomando régimen de Estavudina y Didanosina con un inhibidor de proteasa, no se informó de la exposición a Efavirenz y dos de los niños presentaban lipodistrofia leve concurrente. Un estudio realizado en Uganda sobre la aparición de efectos secundarios en los niños en TAR reportaron un caso de ginecomastia en un niño que tomaba Zidovudina, Lamivudina y Efavirenz, pero la información sobre la edad, el estadio puberal, el seguimiento no se proporcionaron. ${ }^{(19)}$ En este reporte de casos todos los pacientes son varones mayores de 11 años.

En el estudio publicado en Antiviral Therapy, se constató una relación con dos medicamentos antirretrovirales, Efavirenz y Didanosina: $57 \%$ frente a $17 \%$ con la toma de Efavirenz, y $50 \%$ frente al $13 \%$ con Didanosina. En esta serie de casos presentada se observa la aparición de las mismas con un promedio de 8 años después de iniciada la terapia antirretroviral con un esquema que contenía Efavirenz, ninguno de ellos había usado Didanosina. En ese mismo estudio publicado se incluyó un total de 1.304 pacientes que no presentaban ninguna otra causa potencial de ginecomastia. Su frecuencia, confirmada mediante ecografía y/o mamografía fue del $2,3 \%$. Los expertos analizaron las características de la ginecomastia y observaron que ésta era unilateral en el $53 \%$, dolorosa en el $83 \%$, y duró nueve meses en el $73 \%$ de los casos, el $91 \%$ de los cuales remitió sin haber modificado la TARGA. ${ }^{(20)}$ Esta serie reporta la presencia de las dos presentaciones clínicas: unilaterales y bilaterales con leve dolor.
El diagnóstico de los pacientes presentados fue exclusivamente clínico no se realizó la medición de ninguna hormona tales como: testosterona-total y biodisponible- $y$ el índice de testosterona libre, ni se realizó estudios de imágenes. Los estudios de imágenes de forma rutinaria en pacientes con ginecomastia es controversial, ya que algunos autores consideran que la imagen sólo debe realizarse si se sospecha de malignidad. ${ }^{(21)}$ Solo uno de los pacientes presentados en esta revisión fue interconsultado con endocrinología la conclusión de la misma: Ginecomastia inducida por medicamentos.

El tratamiento de la ginecomastia asociada a TARGA incluye varias opciones, hay pocos estudios con pequeños número de casos que abordan esta cuestión y, por tanto, no existen tratamientos definitivos aún. (22) La resolución espontánea puede ocurrir. A veces, la interrupción de un medicamento antirretroviral puede revertir la ginecomastia. ${ }^{(23)} \mathrm{El}$ uso de testosterona es seguro y eficaz tanto por vía transdérmica o por vía intramuscular así como el gel de dihidrotestosterona percutánea ( $5 \mathrm{~g}$ diaria una vez al día durante 1-3 meses) se pueden utilizar. ${ }^{(24)}$

Algunos estudios han demostrado éxito con el uso de Tamoxifén 10-20 mg dos veces al día, sobre todo en los casos de reciente aparición. ${ }^{22,25,26)}$ La cirugía también ha sido considerada solo el $10 \%$ de los casos especialmente por motivo estético. ${ }^{27,28)}$ En los pacientes de esta serie se decidió el retiro de efavirenz después de un periodo de observación en dos de los cuatro y retiro inmediato en los otros dos, motivados exclusivamente por las repercusiones psicológi- 
cas observadas en ellos. Resolución del cuadro en uno de los cuatro pacientes presentados, cabe mencionar que en el más antiguo de ellos.
En resumen creemos que la ginecomastia es un efecto adverso que debe sospecharse y vigilarse en los pre-púberes cuyo esquema de tratamiento antirretroviral tenga incluido el efavirenz.

\section{BIBLIOGRAFÍA}

1. Deepinder F, Braunstein GD. Drug-induced gynecomastia: an evidence-based review. Expert Opin Drug Saf. [Internet] 2012[Citado 25 de octubre 2014]; 11(5): 779-95. Disponible en: http://www. ncbi.nlm.nih.gov/pubmed/22862307

2. Nordt CA, Di Vasta Amy D. Gynecomastia in adolescents. Curropin Opinion in Pediatr. [Internet] 2008[Citado 22 de octubre 2014];20(4):375-382.

Disponible en: http://journals.Iww.com co-pediatrics/Abstract/2008/08000/Gy necomastia_in_adolescents.3.aspx

3. Braunstein GD. Gynecomastia. N Engl J Med. [Internet] 2007[Citado 13 de octubre 2014]; 357:1229-1237. Disponible en: http://www.nejm.org/doi/full/10.1056 /NEJMcp070677

4. Comité Internacional de Endocrinología. Enfoque práctico del manejo de la ginecomastia. Seis preguntas que debe responderse el pediatra ante un paciente con ginecomastia. Arch Argent Pediatr. [Internet] 2011[Citado 20 de octubre 2014]; 109(4):365-368. Disponible en: http://www.scielo.org.ar/scielo.php?script $=$ sci_arttext $\&$ pid $=$ S0325-00752 011000400020\&lang $=p t$
5. Goldman RD. Drug-induced gynecomastia in children and adolescents. Can Fam Physician. [Internet] 2010[Citado 15 de octubre 2014];56(4): 344-45. Disponible en: http://www.ncbi.nlm.nih. gov/pubmed/20393092

6. Elav-Bachar R, Phillip M, Aurbach-Klipper Y, Lazar L. Prepubertal gynaecomastia: aetiology, course and outcome. Send to: Clin Endocrinol (Oxf). [Internet] 2004[Citado 22 de octubre 2014]; 61(1): 55-60. Disponible en: http://www.ncbi. nlm.nih.gov/pubmed/15212645

7. Pachon Díaz J, Pujol De La llave E. La infección por el VIH: Guía práctica. $2^{\mathrm{a}}$ Edición. España 2 ed 2003; 33: 405-409

8. Oventon Turner. Review of Bone Posters at CROI 2008. 15th CROI Conference on Retroviruses and Opportunistic Infections Boston 2008, febrero 2-3. Actas de la. Conference Reports for NATAP. Boston 2008. http://www.natap.org/2008/CROI/croi_126.htm

9. Secretaria de salud Manual de Atención Integral al Adulto y al adolescente con VIH. Honduras. C.A. 2013. 
10. Deeks SG, Barbour JD, Martin JN, Swanson MS, Grant RM. Sustained CD4+ T Cell Response after Virologic Failure of Protease Inhibitor-Based Regimens in Patients with Human Immunodeficiency Virus Infection. J Infect Dis. [Internet] 2000 [Citado 29 de octubre 2014]; 181(3): 946-53.

Disponible en: http://www.ncbi.nlm.nih. gov/pubmed/10720517

11. Piroth L, Grappin M, Petit JM, Buisson M, Duong M, Chavanet P, et. al. Incidence of gynaecomastia in men infected with HIV and treated with highly active antiretroviral therapy. Scand J. Infect Dis. [Internet] 2001[Citado 18 de septiembre 2014]; 33(7):559-560.

Disponible en: http://www.ncbi.nlm.nih. gov/pubmed/11515774.

12. Van Ramshorst MS, Kekana M, Struthers HE, Mclntyre JA, Peters RP. Efavirenz-induced gynecomastia in a prepuberta girl with human immunodeficiency virus infection: a case report. BMC Pediatrics. [Internet] 2013[Citado 13 de octubre 2014];13:120. Disponible en: http://www.ncbi.nlm.nih.gov/pmc/arti cles/PMC3751361/

13. Caso JA, Prieto Jde M, Casas E, Sanz J. Gynecomastia without lipodystrophy syndrome in HIV-infected men treated with efavirenz.AIDS. [Internet] 2001 [Citado 11 de octubre 2014]; 15(11): 1447-1448. Disponible en: http://www. ncbi.nlm.nih.gov/pubmed/11504970

14. Mercie $P$, Viallard JF, Thiébaut $R$, Faure I, Rispal P, Leng B, et. Al. Efavirenz-as sociated breast hypertrophy in HIV-infection patients. AIDS. [Internet] 2001 [Citado 13 de octubre 2014]; 15(1): 126-129. Disponible en: http://www. ncbi.nlm.nih.gov/pubmed/11192857

15. Benveniste O, Simon A, Herson S .Successful percutaneous dihydrotestosterone treatment of gynecomastia during highly active antiretroviral therapy, four cases and a review of the literature. Clinical Infect Dis. [Internet] $2001 \mathrm{Sept}$ 15[Citado 12 de octubre 2014]; 33(6): 891-3. Disponible en: http://www.ncbi. nlm.nih.gov/pubmed/11512095

16. Sikora MJ, Rae JM, Johnson MD, Desta $Z$ : Efavirenz directly modulates theoestrogen receptor and induces breast cancer cell growth.HIV Med. [Internet] 2010[Citado 10 de octubre 2014]; 11(9): 603-607. Disponible en: http://www.ncbi.nlm.nih.gov/pubmed/20408889

17. Manfredi R, Calza L, Chiodo F: Another emerging event occurring duringHIV infection treated with any antiretroviral therapy: frequency and role of gynecomastia. Infez Med. [Internet] 2004 [Citado 09 de octubre 2014];12(1):51-59. Disponible en: http://www.ncbi.nlm.nih. gov/pubmed/15329529

18. Manfredi R, Calza L, Chiodo F: True gynecomastia in congenitally HIV-infected children treated with antiretroviral agents. J Chemother. [Internet] 2004[Citado 12 de octubre 2014]; 16(3): 303-305. Disponible en: http://www. ncbi.nlm.nih.gov/pubmed/15330330 
19. Tukei VJ, Asiimwe A, Maganda A, Atugonza R, Sebuliba I, Bakeera-Kitaka $S$, Et al. Safety and tolerability of antiretroviral therapy among HIV-infected children and adolescents in Uganda. J Acquir Immune Defic Syndr. [Internet] 2012 [Citado 13 de octubre 2014]; 59(3):274- 280. http://www.ncbi.nlm. nih.gov/pubmed/22126740

20. Calmy A, Hirschel B, Cooper DA, Carr A. A new era of antiretroviral toxicity. Antivir Ther. [Internet] 2009[Citado 14 de octubre 2014];14(2):165-79. Disponible en: http://www.ncbi.nlm.nih.gov/pubmed/ 19430091

21. Narula HS, Carlson HE. Gynaecomastia. Endocrinol Metab Clin North Am. [Internet] 2007[Citado 13 de octubre 2014] Jun; 36(2):497-519. Disponible http://www.ncbi.nlm.nih.gov/pubmed/ 17543732

22. García-Benayas T, Blanco F, Martín-Carbonero L, Valencia E, Barrios A, González-Lahoz J, et al. Gynaecomastia in HIV infected patients receiving ARV treatment. AIDS Res Hum Retroviruses. [Internet] 2003 [Citado 17 de octubre 2014] Sep;19(9):739-41. http:// www.ncbi.nlm.nih.gov/pubmed/145 85204

23. Roberts John R. What Is Immune Restoration Inflammatory Syndrome Resulting From Highly Active Antiretroviral Therapy (HAART). [Internet] Medscape. 2001[Citado 20 de octubre 2014]; 3(1). Disponible en: http://www.medscape. com/viewarticle/413384
24. Jover F, Cuadrado JM, Roig P, Rodríguez M, Andreu L, Merino J. Efavirenz-associated gynaecomastia .Report of 5 cases and review of the literature. Breast J. [Internet] 2004[Citado 21 de octubre 2014]; 10(3):244-6. Disponible en: http://www.ncbi.nlm.nih.gov/pubmed $/ 15125753$

25. Kegg S, Lau R. Tamoxifen in antiretroviral-associated gynaecomastia. Int $\mathrm{J}$ STD AIDS. [Internet] 2002[Citado 23 de octubre 2014]; 13(8):582-583. Disponible en: http://www.ncbi.nlm.nih.gov/pub med/12194746

26. Debra Meerkotter. Gynaecomastia associated with highly active antiretroviral therapy (HAART). J Radiol Case Rep. [Internet] 2010[Citado 25 de octubre 2014]; 4(7): 34-40. Disponible en: http://www.ncbi.nlm.nih.gov/pmc/arti cles/PMC3303362/

27. Devoto C Enzo, Madariaga A Marcia, Lioi C Ximena, Mardones Nelly. Terapia médica de la ginecomastia con tamoxifeno. Influencia del volumen y duración de la ginecomastia en el resultado terapéutico. Rev. méd. Chile [Internet]. 2007 Dic [citado 2016 Ene 22]; 135 (12): 1558-1565. Disponible en: http:// www.scielo.cl/scielo.php?script=sci_arttext\&pid $=0034-98872007001200009 \&$ I ng=es. $\quad$ http://dx.doi.org/10.4067/S00 34-98872007001200009.

28. Figueroa AS, Romero FV. Patología quirúrgica de mama en pacientes pediátricos. Rev Peru Ginecol Obstet. 2012; 58:177-182 